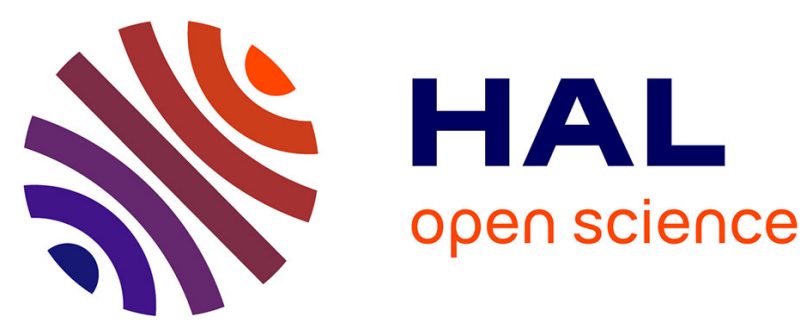

\title{
Effects of a Diverging Cup on Swirl Number, Flow Pattern and Topology of Premixed Flames
}

Arthur Degenève, Paul Jourdaine, Clément Mirat, Jean Caudal, Ronan Vicquelin, Thierry Schuller

\section{- To cite this version:}

Arthur Degenève, Paul Jourdaine, Clément Mirat, Jean Caudal, Ronan Vicquelin, et al.. Effects of a Diverging Cup on Swirl Number, Flow Pattern and Topology of Premixed Flames. ASME Turbo expo 2018, Jun 2018, Lillestrom, Norway. 10.1115/gt2018-76152 . hal-01825590

\section{HAL Id: hal-01825590 \\ https://hal.science/hal-01825590}

Submitted on 28 Jun 2018

HAL is a multi-disciplinary open access archive for the deposit and dissemination of scientific research documents, whether they are published or not. The documents may come from teaching and research institutions in France or abroad, or from public or private research centers.
L'archive ouverte pluridisciplinaire HAL, est destinée au dépôt et à la diffusion de documents scientifiques de niveau recherche, publiés ou non, émanant des établissements d'enseignement et de recherche français ou étrangers, des laboratoires publics ou privés. 


\title{
EFFECTS OF A DIVERGING CUP ON SWIRL NUMBER, FLOW PATTERN AND TOPOLOGY OF PREMIXED FLAMES
}

\author{
Arthur Degenève $e^{\mathrm{a}, \mathrm{b}, *}$, Paul Jourdaine ${ }^{\mathrm{b}}$, Clément Mirat $^{\mathrm{a}}$, Jean Caudal $^{\mathrm{b}}$, Ronan Vicquelin ${ }^{\mathrm{a}}$, Thierry Schuller ${ }^{\mathrm{a}, \mathrm{c}}$ \\ ${ }^{a}$ Laboratoire EM2C, CNRS, CentraleSupélec, Université Paris-Saclay, 3 rue Joliot Curie 91192 Gif Sur Yvette cedex, France \\ ${ }^{b}$ Air Liquide, Centre de Recherche Paris-Saclay, 1, chemin de la Porte des Loges, Les Loges en Josas BP 126, 78354 Jouy-en-Josas Cedex, France \\ ${ }^{c}$ Institut de Mécanique des Fluides de Toulouse, IMFT, Université de Toulouse, CNRS, Toulouse, France
}

\begin{abstract}
Impact of the diverging cup angle of a swirling injector on the flow pattern and stabilization of technically premixed flames is investigated both theoretically and experimentally with the help of laser diagnostics. Recirculation enhancement with a lower position of the internal recirculation zone and a flame leading edge protruding further upstream in the swirled flow are observed as the injector nozzle cup angle is increased. A theoretical analysis is carried out to examine if this could be explained by changes of the swirl level as the diffuser cup angle is varied. It is shown that pressure effects need in this case to be taken into account in the swirl number definition and expressions for its variation through a diffuser are derived. They indicate that changes of the swirl level including or not the pressure contribution to the axial momentum flux cannot explain the changes observed of the flow and flame patterns in the experiments. The swirl number without the pressure term, designated as pressure-less swirl, is then determined experimentally for a set of diffusers with increasing quarl angles under non-reacting conditions and the values found corroborate the predictions. It is finally shown that the decline of axial velocity and the rise of adverse axial pressure gradient, both due to the cross section area change through the diffuser cup, are the dominant effects that control the leading edge position of the internal recirculation zone of the swirled flow. This in turn is used to develop a model for the change of this position as the quarl angle varies that shows good agreement with experiments.
\end{abstract}

\section{INTRODUCTION}

Providing a rotational motion to the flow leading to the formation of an Internal Recirculation Zone (IRZ) is widely used to ease flame stabilization in high power combustion systems. Despite extensive studies, see for example the pioneering work in [1], the stabilization mechanisms of these flames are still the topic of many recent investigations due to their complex structure and dynamics [2].

The structure of a swirling flame is known to depend largely on the structure of the irrotational jet exhausting the injector $[3,4,5,6]$. The swirl number $[3,4]$, the inlet geometry of the injector $[7,8]$ and the flow confinement $[9,10]$ are the main parameters affecting the flame topology. Heat losses to the chamber walls are also known to alter the structure of the reacting flow $[11,12]$. A central bluff-body $[13,2,14]$ and a diffuser $[13,4,15,2,16,17]$ constitute other widely used elements to enhance the stabilization of swirling flames.

${ }^{*}$ Corresponding author: arthur.degeneve @ centralesupelec.fr
In high power systems, it is however more suitable to operate without any central insert to reduce the thermal stress on the solid components of the injector.

The diverging cup of the injector nozzle, also designated by quarl or diffuser, drastically changes the topology of the flow so as to favor flame stabilization inside the IRZ. Gupta et al.[4] and Vanoverberghe et al. [15] investigated the combination of swirl, quarl and bluffbody to identify and classify the different flow patterns observed under non reacting flow conditions. Increasing the quarl angle enhances the recirculation of mass flow in the IRZ [18, 15], increases its size and lowers its position along the burner axis [13,4] improving flame stabilization. Adding a quarl is often used to improve the operability of a burner over a wider range of flow operating conditions. However, as already noticed in [15], there is still yet a limited number of studies on effects of the quarl for aerodynamically swirl-stabilized flames in setups without bluff-body.

Both quarl and swirl separately provide interesting features to the resulting flow, yet the quarl may al-

June 28, 2018 
ter the value of the swirl number through the evolution of the velocity profiles. Chigier and Beer [13] introduced the swirl number $S=G_{\theta} /\left(R G_{z}\right)$ to characterize the level of swirl of the flow, where $G_{\theta}=$ $\int_{A} \rho r u_{\theta} u_{z} \mathrm{~d} A$ is the axial flux of tangential momentum, $G_{z}=\int_{A}\left(\rho u_{z}^{2}+\left(p-p_{\infty}\right)\right) \mathrm{d} A$ the axial momentum flux and $R$ a characteristic dimension of the injector.

Gupta and Lilley (p. 18 in [4]) have theorized the impact of a change of section on the value of the swirl number. As in the majority of the works [19], they assume the pressure term in $G_{z}$ to be negligible. This approximation leads to the definition of a pressure-less swirl number $\widetilde{S}$ calculated with $\widetilde{G}_{z}=\int_{A} \rho u_{z}^{2} \mathrm{~d} A$. Assuming simplified velocity profiles, they model the impact of a quarl on the swirl number as:

$$
\frac{\widetilde{S_{2}}}{\widetilde{S}_{1}}=\frac{R_{2}}{R_{1}}
$$

where $R_{1}$ and $R_{2}$ are the radius of the diffuser cup inlet and outlet sections. Experiments presented in this work show that Eq. (1) cannot be a substitute for the complex velocity profiles issuing from a swirling injector. This problem has motivated further investigation. Change of the swirl level through a change of the cross section area of the flow passage is here revisited both experimentally and theoretically.

Measuring the swirl number $S$ raises experimental difficulties. As reported in many studies, swirling flows of practical interest are highly turbulent [19], and Reynolds averages of the axial and azimuthal momentum fluxes lead to new contributions associated to turbulent fluctuations $\overline{G_{z t}}=\int_{A}\left(\rho\left({\overline{u_{z}}}^{2}+\overline{u_{z}^{\prime 2}}\right)+\left(\bar{p}-p_{\infty}\right)\right) \mathrm{d} A$ and $\overline{G_{\theta t}}=\int_{A} \rho r\left(\overline{u_{\theta}} \overline{u_{z}}+\overline{u_{\theta}^{\prime} u_{z}^{\prime}}\right) \mathrm{d} A$. In the outer regions of the swirling jet, the mean velocities $\bar{u}_{z}$ and $\bar{u}_{\theta}$ drop to zero whereas the turbulent components $\overline{u^{\prime 2}}{ }_{z}$ and $\overline{u_{z}^{\prime} u^{\prime}}{ }_{\theta}$ due to the recirculating flow pattern remain significant. Similarly, $\bar{p}-p_{\infty}$ is large when compared to $\rho{\overline{u_{z}}}^{2}$ in the outer region of the jets. Hence, measuring $\overline{G_{z t}}$ and $\overline{G_{\theta t}}$ in a turbulent swirling flow requires to probe the velocity field up to the vicinity of the walls where the turbulent and pressure terms are weighted by $r^{2}$ to estimate these integrals.

Some authors $[4,20]$ suggest to integrate by part the axial momentum flux, which brings out the static pressure on the wall. Thanks to wall pressure measurements, Mattingly et al.[20] verified the conservation of axial momentum flux in a tube of constant cross section area. Other authors directly measured the radial distribution of static pressure within the flow with the help of Pitot probes[4, 20]. Chigier et al.[13], Dixon et al.[21]

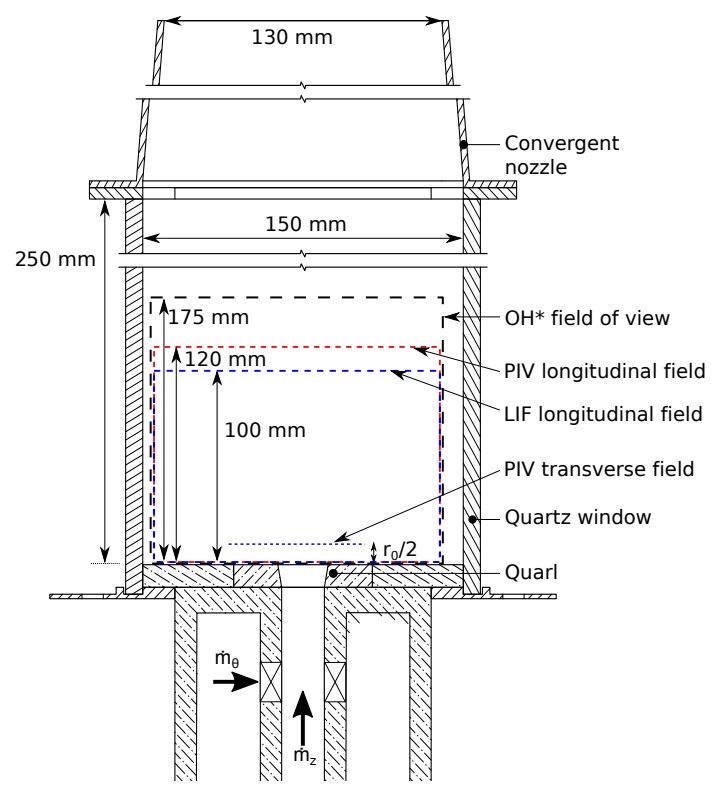

Figure 1: OXYTEC atmospheric test-rig.

and Mahmud et al.[22] found that both $G_{z}$ and $G_{\theta}$ momentum fluxes remain constant within a straight tube provided effects of pressure is included. However, Pitot probes are intrusive devices and smooth out turbulent fluctuations.

These previous investigations show that measuring the swirl number is a difficult task due to the additional contributions from pressure and turbulent fluctuations. Effects of turbulence are discarded in the present work and a theoretical analysis is carried out to estimate the contribution from the pressure term in the swirl level due to changes of the cross section area through an injector. This problem constitutes the first objective of this article. The second objective is to understand how the flow structure and flame stabilization are modified when the angle of the diffuser cup of the injector is changed. An experimental analysis is conducted to isolate effects of the quarl angle, all other parameters remaining fixed. It is shown that measurements of the pressure-less swirl number do not obey to Eq. (1) and this has motivated a further theoretical investigation of the swirl number evolution through a diffuser with the introduction of shape factors. It is finally shown that independently of the definition of the swirl number, changes of the swirl level cannot be used to explain the changes of the average reacting and non-reacting flow fields observed in the experiments when the injector diffuser cup angle widens. This in turn has led to the development of a new model that predicts the evolution of the leading edge position of the internal recirculation zone of a swirling injector when the quarl angle is varied. 
The article is organized as follows. The experimental setup is described in the next section, followed by an analysis of the flame and flow structures in reacting conditions for varying quarl angles. Measurements of swirl number on a pressure-less basis are then carried out under-non reacting conditions. Theory is then pushed forward to include pressure effects and examine the impact of a smooth change of the cross section section area of the injector on the swirl number. Finally, a model is developed to account for the displacement of the position of the IRZ in the combustion chamber when the diverging cup angle is modified.

\section{EXPERIMENTAL SETUP}

The test rig and the optical diagnostics are the same as those used to investigate effects of swirl on the stabilization of technically premixed methane/air flames in a configuration where the injection nozzle is equipped with a diverging cup angle $\alpha=10^{\circ}$ [17]. This setup was also used to compare the stabilization of $\mathrm{CO}_{2-}$ and $\mathrm{N}_{2}$-diluted oxy-methane flames and examine rules for switching between air- to oxy-combustion operating mode with the same injector [23, 17]. Only the main elements of the test rig are briefly described below. The reader is referred to $[23,17,24]$ for more details.

Figure 1 shows a schematic of the Oxytec combustor. The combustion chamber has a square cross section with dimensions $150 \times 150 \mathrm{~mm}^{2}$ and $250 \mathrm{~mm}$ in length. Four quartz windows provide a large optical access to the combustion region. The burnt gases are exhausted to the atmosphere at ambient pressure through a nozzle with an area contraction ratio of 0.8 . The combustion chamber dump plane in contact with the burnt gases is cooled by water circulation. Its temperature is kept constant and equal to $T_{p}=450 \mathrm{~K}$ during all experiments.

Methane and air are mixed within a swirling injector sketched in Fig. 2. The swirling motion is produced by an axial-plus-tangential entry swirl generator where $\dot{m}_{\theta}$ and $\dot{m}_{z}$ are the mass flowrates injected tangentially and axially. Assuming an uniform axial flow profile and a solid body rotation for the azimuthal velocity, a geometrical swirl number $\mathrm{S}_{0}$ can be defined at the injector outlet [4]:

$$
\mathrm{S}_{0}=\frac{\pi}{2} \frac{H r_{0}}{N l L} \frac{1}{1+\dot{m}_{z} / \dot{m}_{\theta}}
$$

where $H$ is the distance separating the tangential injection channels from the burner axis, $r_{0}$ the injector radius and $l$ and $L$ the width and the height of the $N$ tangential injection channels. This device was designed to produce geometrical swirl numbers ranging from $S_{0}=0$ to
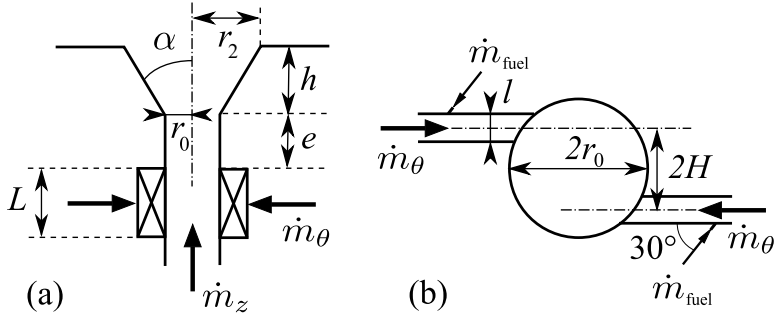

Figure 2: Sketch of the injector. (a) Axial cut. (b) Transverse cut through the swirler.

1.75 with $N=2$ slits. More details on the fuel injection system are given in [24].

The methane/air mixture leaves the swirler through a $r_{0}=10 \mathrm{~mm}$ cylindrical channel and flows into the combustion chamber through an end piece equipped with a diffuser with a variable cup angle $\alpha$. The height of the diffuser cup is $h=10 \mathrm{~mm}$. Partially premixed conditions are achieved at the injector outlet at $z / r_{0}=0$ [17] and fully premixed conditions were confirmed by Large Eddy Simulations at $z / r_{0}=0.5$ in a region where the flame leading edge is stabilized for most operating conditions [24].

Care was taken to wait for thermal equilibrium of the chamber solid components before making measurements [25]. $\mathrm{OH}^{*}$ chemiluminescence images are used to investigate the mean structure taken by the flames. OH Planar Laser Induced Fluorescence $(\mathrm{OH}-$ PLIF) snapshots are used to determine the probability of presence of the flame front in the axial plane of the test-rig. A set of 1500 images is taken to deduce the probability of presence of the hot burnt gases. The gradient of these images is then used to detect the flame front between the fresh gases and the hot burnt gases. Averages of these images yield the probability of presence of the flame front. A series of tests were made to check the statistical convergence of the data and the sensitivity of the results to the threshold level used to detect the flame front. The reader is referred to [24] for more details on the post-processing.

These experiments are completed by Particle Image Velocimetry (PIV) measurements in cold and hot flow conditions in the axial and different transverse planes above the injector. PIV and OH-PLIF are here combined to reveal the mean structure taken by the flow and flame produced by the axial-plus-tangential swirler. Two component Laser Doppler Velocimetry (LDV) measurements have also been carried out to determine the axial and tangential velocity components of the non reacting swirling flow at the injector outlet with a high degree of accuracy required to determine the (pressure-less) swirl number. The diagnostics, the 


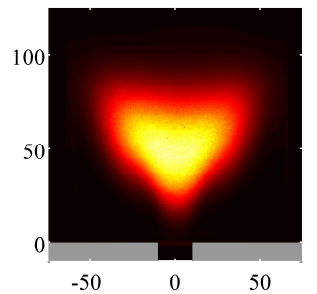

(a) $\alpha=0^{\circ}$

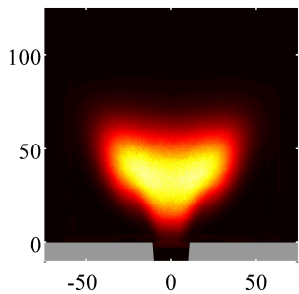

(b) $\alpha=5^{\circ}$

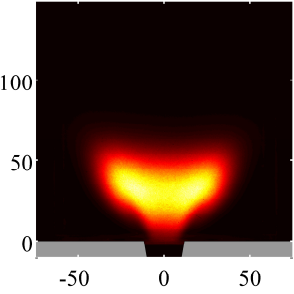

(c) $\alpha=10^{\circ}$

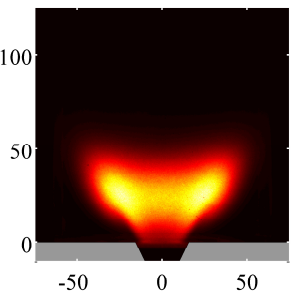

(d) $\alpha=30^{\circ}$

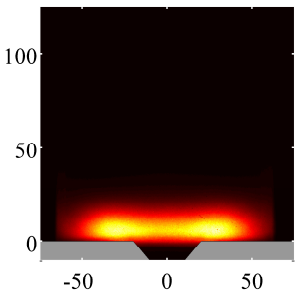

(e) $\alpha=45^{\circ}$

Figure 3: $\mathrm{OH}^{*}$ intensity distribution as a function the diverging cup angle $\alpha$. Grey elements indicate solid components of the combustor. Dimensions are in millimeters.

tests made and the different post-processing techniques are fully described in $[17,24]$.

All experiments presented in this work are conducted at the equivalence ratio $\phi=0.95$ for a thermal power $P=13 \mathrm{~kW}$ corresponding to a Reynolds number $\mathrm{Re}=18000$ based on the injection tube diameter $2 r_{0}=20 \mathrm{~mm}$ and the bulk temperature $T_{u}=293 \mathrm{~K}$. The geometrical swirl number calculated with Eq. (2) is also kept constant and equal to $S_{0}=0.85$. Note that effects of the diverging cup are not taken into account in this definition of the swirl number.

\section{FLAME AND FLOW STRUCTURES}

Before examining the flame structure, it is worth attempting a dimensional analysis of the main parameters controlling the flame shape. The height of the combustor being fixed, the main important parameters identified in the scientific literature are the injection Reynolds number Re [26], the quarl angle $\alpha[18,15]$, the swirl number $S[3,4]$ and the confinement ratio $C_{r}=W^{2} /\left(\pi r_{2}^{2}\right)[10,9]$, where $W=150 \mathrm{~mm}$ is the width of the combustion chamber and $r_{2}$ is the nozzle radius at the diffuser cup outlet. The quarl angle varies here from $\alpha=0$ to $45^{\circ}$ and $18 \leq C_{r} \leq 72$. As a consequence, the injector of the Oxytec test-rig operates according to [9] in the free-jet regime. This regime is typical of a swirling jet issuing into unconfined atmosphere and of systems with sidewalls aways from the injector nozzle.

Figure 3 shows the distribution of the $\mathrm{OH}^{*}$ spontaneous light emission for different quarl angles $\alpha$, all other geometrical and flow parameters remaining constant. With a straight injector, $\alpha=0^{\circ}$, the flame is lifted in a V-shape above the burner with a flame leading edge far from the burner exit despite the large swirl level $S_{0}=0.85$ imparted to the flow. As $\alpha$ increases from $5^{\circ}$ to $30^{\circ}$, the flame widens in the transverse direction, shrinks in the axial direction and its leading edge moves further upstream towards the injector. For larger values $\alpha \geq 45^{\circ}$, the flame suddenly flattens and the combustion reaction takes place in the boundary layer close to the combustor dump plane. The flame takes in these conditions a torus shape in the so-called wall jet regime [4, 13], also referred as Coanda stabilized flame [15].

The same experiments were repeated at a lower swirl number $S_{0}=0.75$ in [17], wherein a more detailed study is carried out on the influence of the swirl level on the flame topology. The same observations were made. Increasing the quarl angle moves the flame leading edge upstream, reducing the flame length and widening its shape in the radial direction. These observations are common to many studies conducted with different types of swirling injectors in premixed and non-premixed combustion modes with gaseous or liquid fuel injections $[4,15]$.

Further analysis is carried out by examining the structure of the flow field and flame in an axial plane. Figure 4 shows on the top the probability of presence of the flame front superimposed to the velocity field obtained by PIV in reacting conditions for different diffuser cup angles, all other parameters remaining fixed. The grey countors represent the position where the flame front is present $20 \%$ (inner contour) and $10 \%$ (outer contour) of the time. The position of the IRZ is represented by the black contour. The position of the Outer Recirculation Zones (ORZ) is not reproduced in this figure. The bottom images show the same velocity fields on a slightly zoomed field of view together with the magnitude $|\bar{v}|=\left(\bar{u}_{z}^{2}+\bar{u}_{x}^{2}\right)^{1 / 2}$ of the velocity vectors $\overline{\mathbf{v}}=\bar{u}_{z} \mathbf{e}_{z}+\bar{u}_{x} \mathbf{e}_{x}$ represented by the colored scale. The black line delineates the position where the axial velocity $\bar{u}_{z}=0$ is null, i.e. the boundary of the IRZ. The contours of zero axial velocity delineating the ORZ are not represented here.

For a straight injection nozzle $\alpha=0^{\circ}$, the V-shaped flame features a leading edge front located along the burner axis at a distance $z_{f} / r_{0}=1.8$ above the injector outlet, identified here as a probability of presence 

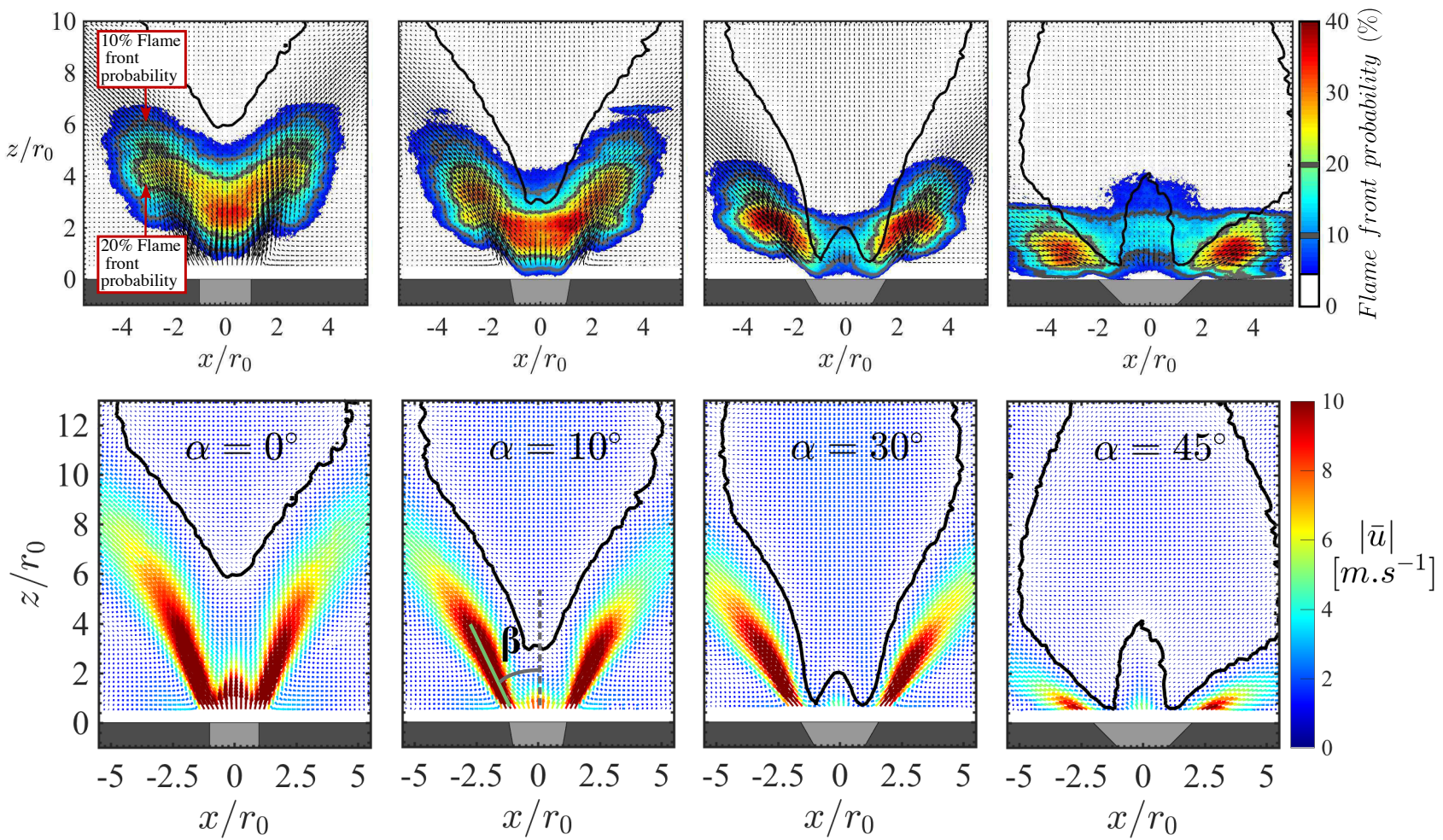

Figure 4: Top: Probability of presence of the flame front deduced from OH-PLIF measurements in an axial plane with the overlaid velocity fields. The grey lines delineate the positions where the flame front is present $20 \%$ and $10 \%$ of the time. Bottom: Velocity field colored by the velocity magnitude $|\bar{u}|=\left(\bar{u}_{z}^{2}+\bar{u}_{x}^{2}\right)^{1 / 2}$ obtained by PIV. The black contour delineates the position of the IRZ where the axial velocity $\bar{u}_{z}$ is zero.

of the flame front equal to $p=20 \%$. The stagnation point corresponding to the leading edge of the IRZ $z_{S P} / r_{0}=5.9$ is also located along the burner axis and lies far away from the flame. Note also that the maximum probability of presence of the flame front does not exceed in this case $p<30 \%$ highlighting the strong intermittency of the combustion process, a characteristic of turbulent swirling flames stabilized far away from the injector outlet. It can be noticed that the combustion reaction takes also place between the ORZ and the outer swirling jet shear layer. The flame takes in this case intermittently an M-shape, with rapid transitions back to its V-shape. The probability of presence of the M-shape structure remains small $p<20 \%$ due to the high dump ratio of the combustor leading to high thermal losses in the ORZ [12]. The ORZ is too cold to sustain combustion between the ORZ and the outer shear layer of the swirling jet.

When the injection nozzle is equipped with a diffuser cup angle $\alpha=10^{\circ}$, the flame still mainly features a Vshape, but lies closer to the injector outlet with a leading edge front at $z_{f} / r_{0}=1.0$. The probability of presence of the flame front increases above $p \geq 20 \%$ with a large fraction with $p \sim 35 \%$ over most of the flame volume. The flame leading edge front now lies on both sides of the burner axis, above the regions featuring the lowest axial velocities at $|x| / r_{0}=0.8$. This is due to the peculiar structure of the jet flow at the nozzle outlet produced by this axial-plus-tangential injector. The flame leading edge preferentially lies in a region comprised between the burner axis where the axial velocity reaches a local maximum and the inner shear layer of the swirling jet at $|x| / r_{0}=1.2$ where the velocities are the highest. The trace of the statistical distribution of the leading edge reaction layer follows the axial velocity profile and also takes a smoothed but discernable $\mathrm{W}$-shape. One may also note that the leading edge of the IRZ at $z_{S P} / r_{0}=3.0$ is no longer located along the burner axis. The flame front probability of presence at the interface between the ORZ and the outer shear layer of the swirling jet has slightly increased with values $p>20 \%$, meaning that the probability to find the M-shaped flame distribution has slightly increased compared to injection with the straight injection tube $\left(\alpha=0^{o}\right)$. 


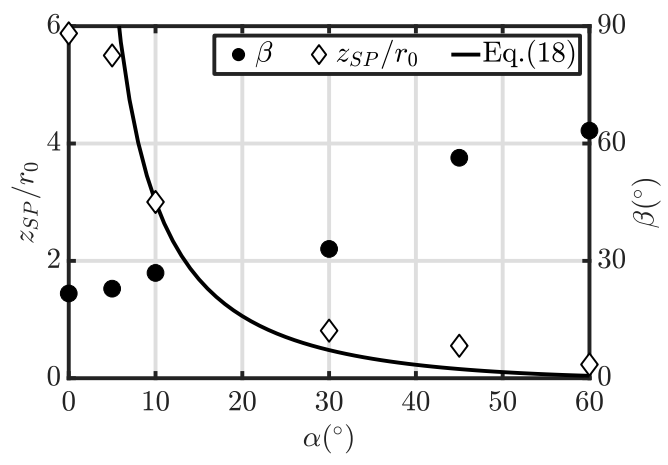

Figure 5: Angle $\beta$ (black disks) of the swirling jet flow at the injector outlet and position of the IRZ leading edge stagnation point $z_{S P} / r_{0}$ measured (empty diamonds) and predicted by Eq. ( 19) (continuous line) as function of the diffuser cup angle $\alpha$.

When the diffuser cup angle is further increased to $\alpha=30^{\circ}$, the flame now switches intermittently between a V-shape and an M-shape with about the same probability. The IRZ leading edge moves very close to the maximum probability of presence of the flame front with a leading edge position at $z_{S P} / r_{0}=0.8$ and is offaxis by $|x| / r_{0}=1.0$. Note that the stagnation point of the IRZ along the burner axis lies much further away at $z_{S P} / r_{0}=1.8$. The flame leading edge position also lies off-axis at the same distance $z_{f} / r_{0}=0.8$ as the leading edge of the IRZ, but is pushed radially away from the burner axis at $|x| / r_{0}=1.5$. The main difference with results for a cup angle $\alpha=10^{\circ}$ is that for $\alpha=30^{\circ}$ the IRZ now protrudes far upstream close to the injector outlet. This protruding IRZ shrinks the size of the flame in the axial direction with almost no reaction left in the central region of the flow and pushes the combustion zone toward the side of the burner. The probability of presence of the flame front remains lower than $p<15 \%$ along the burner axis. The combustion reaction is now essentially concentrated in the internal and external shear layers of the flow between the IRZ and ORZ. On average the trace of the distribution of the leading edge of the flame reaction layer lies again in the zones of low axial velocities at $|x| / r_{0}=1.0$ and takes a $\mathrm{W}$-shape. This $\mathrm{W}$-shape is now more apparent than for the case with $\alpha=10^{\circ}$.

For a diffuser cup angle $\alpha=45^{\circ}$, the flame takes a torus shape stabilized close to the dump plane of the injector in a wall jet regime. This flow regime is characterized by the disappearance of the ORZ and a predominant IRZ occupying almost all the combustion chamber except the central region of the flow close to the injector outlet. The probability of presence of the flame front increases now up to values $p \sim 40 \%$ and the reaction mainly takes place along the arms of the swirling jet.

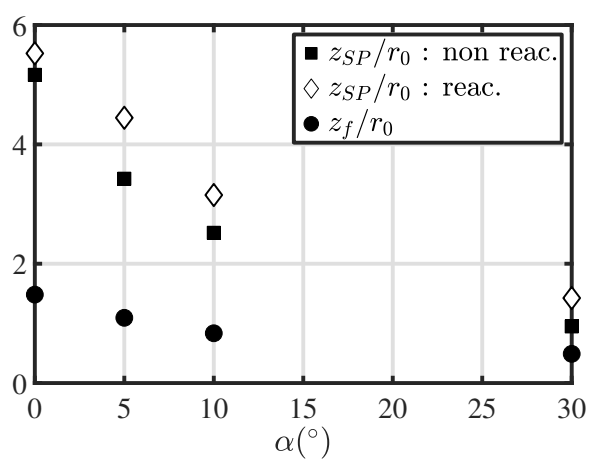

Figure 6: IRZ leading edge position $z_{S P} / r_{0}$ in non-reacting (black squares) and reacting (empty diamonds) flow conditions, and flame leading edge position $z_{f}$ (black disks) as a function of the injector diffuser cup angle $\alpha$.

In this wall jet regime, the IRZ doesn't move further upstream, but grows bigger in the transverse direction because the axial velocity at the burner outlet is high enough to avoid flashback. This feature is a specificity of the axial-plus-tangential swirler used in this study allowing independent control of the axial and tangential mass flowrates injected in the burner. This is used to prevent flashback $[17,27]$.

Effects of the quarl angle are further analyzed by measuring the jet opening angle $\beta$ of the swirling jet. This angle represented in the second image at the bottom in Fig. 4 is defined as the angle between the vertical axis and the line of maximum velocity reached by the jet flow over the first $10 \mathrm{~mm}$ above the injector outlet. The evolution of $\beta$ is plotted in Fig. 5 as a function of the diffuser cup angle $\alpha$. The position of the stagnation point $z_{S P}$ defined as the lowest axial position of the IRZ is also represented in this figure. The angle $\beta$ linearly increases with $\alpha$ below $\alpha \leq 30^{\circ}$. It then changes abruptly for $30^{\circ}<\alpha<45^{\circ}$ when the jet switches to the wall jet regime. This analysis confirms that the swirling jet angle $\beta$ regularly increases like the angle $\alpha$ of the diffuser cup as long as the swirling jet flow lies in the free jet regime.

The flow field is now analyzed by comparing measurements in reacting and non-reacting conditions. Data gathered under non-reacting conditions are not shown here (see [17]). In these experiments, the bulk flow velocity is compensated for the absence of fuel in the nonreacting conditions. Figure 6 represents the IRZ leading edge position $z_{S P} / r_{0}$ in reacting (black squares) and non-reacting (empty diamonds) conditions. The position of the flame leading edge front $z_{f} / r_{0}$ (black disks) is also plotted. The combustion reaction slightly alters the position of the IRZ. Acceleration of the burnt 
gases due to thermal expansion pushes the IRZ a bit further downstream from the injector outlet, but differences for $z_{S P} / r_{0}$ between cold flow and hot flow results remain small. This figure also confirms that the flame leading edge $z_{f} / r_{0}$ always lies upstream the IRZ leading edge $z_{S P} / r_{0}$ with and without the combustion reaction. Consequently, measurements of the flow in non-reacting conditions allow to infer the position of the leading point of the IRZ and the flow regime of the swirling jet with good confidence.

\section{SWIRL NUMBER MEASUREMENTS}

Laser Doppler Velocimetry measurements are carried out in non-reacting conditions to determine the three components of the velocity field at the injector outlet. In these experiments, the bulk flow velocity in the injector is compensated for the absence of fuel. Results for the mean (a)-(b) and rms (c)-(d) velocities are presented in Fig. 7 for quarl angles $0^{\circ} \leq \alpha \leq 30^{\circ}$. As the pressure field could not be determined, the axial momentum $G_{z}$ is approached by its pressure-less equivalent $\widetilde{G}_{z}$, yielding the pressure-less swirl number $\widetilde{S}$ :

$$
\widetilde{S}=\frac{\int_{A} r \bar{u}_{z} \bar{u}_{\theta} \mathrm{d} A}{r_{2} \int_{A} \bar{u}_{z}^{2} \mathrm{~d} A}
$$

where $r_{2}$ is the diffuser outlet radius and $A$ denotes the integration area over the entire cross section of the combustion chamber.

As both $\bar{u}_{z}$ and $\bar{u}_{\theta}$ drop to zero out of the swirling jet, it has been verified that the measured value for $\bar{S}$ does not depend on the choice of the size of the integration area $A$. When measured at $z / r_{0}=0.5$, the axial and tangential velocities are always null at the end of the probed volume. These conditions could not be met further downstream $z / r_{0}>0.5$ or for large quarl angles $\alpha \geq 45^{\circ}$ due to the limited optical access through the combustion chamber.

One reminds that the geometrical swirl number $S_{0}=$ 0.85 is kept constant for all explored cases. The measured values for $\widetilde{S}$ are reported in Tab. 1 for the different quarl angles. It was not possible to perform exploitable measurements of the swirl number in the wall jet regime for $\alpha=45^{\circ}$. It is found that the swirl number $\widetilde{S}$ remains roughly unaltered when the diverging cup angle is varied between $0 \leq \alpha \leq 30^{\circ}$ with the axial-plus-tangential swirler used in this study :

$$
\widetilde{S}_{R_{2}} / \widetilde{S}_{R_{1}} \simeq 1
$$
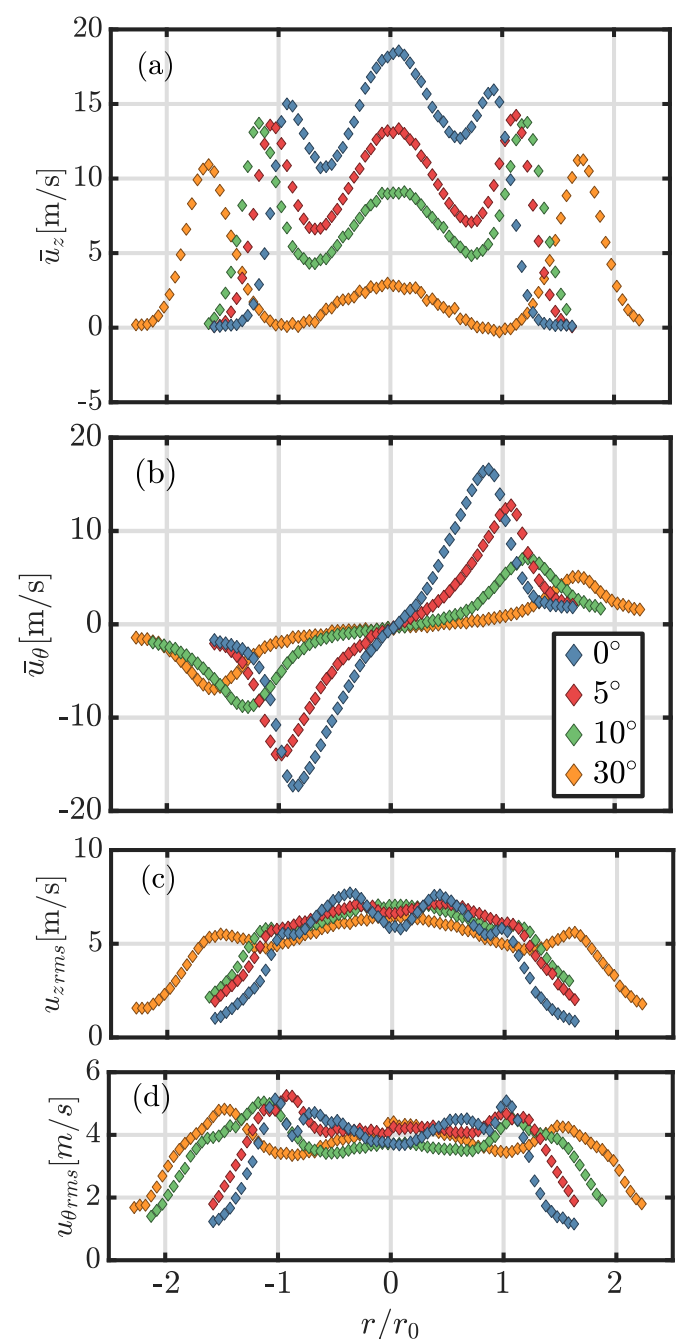

Figure 7: Laser Doppler Velocimetry measurements of the cold swirling flow for $\alpha=0,5,10$ and $30^{\circ} . S_{0}=0.85, \mathrm{Re}=18000$. (a) Mean axial velocity. (b) Mean azimuthal velocity. (c) rms axial velocity fluctuation. (d) rms azimuthal velocity fluctuation.

Table 1: Measured Swirl numbers $\widetilde{S}$ for $\mathrm{Re}=18000$ and velocity profiles at $z / r_{0}=0.5$ for different quarl angles $\alpha$.

\begin{tabular}{|c|c|c|c|c|}
\hline$\alpha\left[^{\circ}\right]$ & 0 & 5 & 10 & 30 \\
\hline$\widetilde{S}$ & 0.78 & 0.72 & 0.78 & 0.74 \\
\hline
\end{tabular}

where $R_{2}$ and $R_{1}$ stand for the outlet radius $r_{2}$ of two different diffusers. This result is at variance with the simplified model Eq. (1) from Gupta and Lilley [4] yielding an increasing swirl level as the quarl angle $\alpha$ increases.

It also results that the evolution of $\widetilde{S}$ with respect to the widening of the quarl angle cannot be used to interpret the flame topologies seen in Figs. 3 and 4. Increasing the swirl level, with a swirl number calculated on 
a pressure-less basis, shortens the flame and shifts the recirculation zone further upstream [17]. The Oxytec burner exhibits here that enlarging the quarl angle does not alter the pressure-less swirl number and still lowers the position of the IRZ. At this point, either the common assumptions made to measure the swirl number are inadequate to configurations featuring a diverging quarl or the swirl number is not the relevant quantity to investigate the behavior of an injector when the diffuseur cup angle is modified.

One may first wonder if this difference could be attributed to effects of turbulence that would alter the swirl level between the inlet and outlet of the diffuser cup. As mentioned previously, the rms velocity fluctuations plotted in Fig. 7(c)-(d) do not drop to zero away from the burner axis and these data cannot be used to make reliable estimates of the swirl number. However, it appears that the level of turbulence is high for the four flows produced by the different quarls. With the $30^{\circ}$ quarl, the rms velocities even surpass the mean values over a large section of the combustion chamber. Finally, the rms values reached by the axial and azimuthal velocities barely change when the quarl angle varies. One can therefore hypothesize that taking into account the contributions from the turbulent velocity fluctuations in the swirl number estimates would lead to reduced variations of the swirl number when the quarl angle is varied.

As stated in the introduction, the experiments from Chigier and Beer [13] and Mahmud et al. [22] show that the momentum fluxes $G_{\theta}$ and $G_{z}$ remain constant when the static pressure is included in the calculation of $G_{z}$. A theoretical analysis is developed in the next section to shed further light on this issue.

\section{THEORETICAL ANALYSIS}

The analysis is made by starting from first principles for a constant density flow. As sketched in Fig. (8), a fixed control volume is considered with a cross section inlet $A_{1}$ and a cross section outlet $A_{2}$ oriented along the vertical axis $\mathbf{e}_{z}$. This volume is bounded on its lateral side by an impermeable boundary over a surface area $\Sigma$. Rotational symmetry of the flow and of the control volume boundaries are assumed. For a steady, inviscid, turbulence free and gravity free flow, the projection of the axial and azimutal momentum balances along the

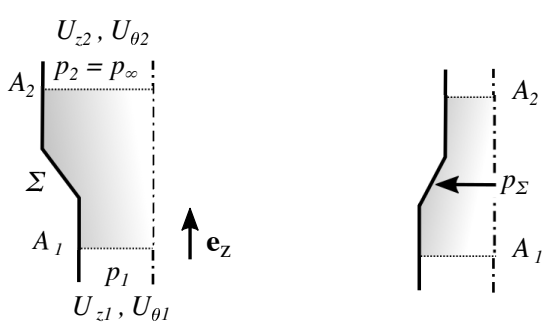

Figure 8: Notations for the theoretical analysis. Right: the swirl number increases in a converging nozzle because $p_{\Sigma}>p_{\infty}\left(C_{F}<0\right)$. Left: the swirl number decreases in a diffuser because $p_{\Sigma}<p_{\infty}\left(C_{F}>0\right)$ provided the pressure loss is not too large.

vertical axis yields :

$$
\left\{\begin{array}{l}
\int_{A_{2}}\left(\rho u_{z}^{2}+p\right) \mathrm{d} A-\int_{A_{1}}\left(\rho u_{z}^{2}+p\right) \mathrm{d} A=-\int_{\Sigma} p \mathbf{n} \cdot \mathbf{e}_{z} \mathrm{~d} A \\
\int_{A_{2}} \rho r u_{\theta} u_{z} \mathrm{~d} A-\int_{A_{1}} \rho r u_{\theta} u_{z} \mathrm{~d} A=0
\end{array}\right.
$$

where $\mathbf{n}$ is the external normal unit vector to the control volume boundary.

The quantity $p_{\infty} \int_{A} \mathbf{n} \cdot \mathbf{e}_{z} \mathrm{~d} A=0$ is subtracted from the momentum balance, where $p_{\infty}$ corresponds to the ambiant pressure, which is taken constant. One is left with :

$$
\left\{\begin{array}{l}
G_{z 2}-G_{z 1}=F_{z} \\
G_{\theta 2}-G_{\theta 1}=0
\end{array}\right.
$$

where $G_{z j}$ and $G_{\theta j}$ are respectively the axial and tangential momentum flux projections through the cross sections $A_{j}$ with $j=1,2$ :

$G_{z j}=\int_{A_{j}}\left(\rho u_{z}^{2}+\left(p-p_{\infty}\right)\right) \mathrm{d} A \quad, \quad G_{\theta j}=\int_{A_{j}} \rho r u_{\theta} u_{z} \mathrm{~d} A$

and $F_{z}$ denotes the axial force exerted by the solid boundaries on the flow. Due to the rotational symmetry, this force is oriented along the vertical axis:

$$
F_{z}=-\int_{\Sigma}\left(p-p_{\infty}\right) \mathbf{n} \cdot \mathbf{e}_{z} \mathrm{~d} A
$$

The choice of $p_{\infty}$, albeit indisputable in an unconfined jet in still air, can be debated when the jet flows into a combustion chamber, where the mean pressure differs from the atmospheric pressure. In this theoretical study, confinement is not taken into account, so that $p_{\infty}=p_{2}$, the pressure at the diffuser outlet.

One designates by $C_{F}=F_{z} / G_{z 1}$ the pressure force made dimensionless by the axial momentum flux in section 1. The swirl number is also defined as $S=$ $G_{\theta} /\left(R G_{z}\right)$, where $R$ is the radius of the cross section 
area of interest. The evolution of the swirl number $S$ between an inlet with section $A_{1}$ and and outlet with section $A_{2}$ can thus be expressed as:

$$
\frac{S_{2}}{S_{1}}=\frac{R_{1}}{R_{2}} \frac{1}{1+C_{F}}
$$

Assuming that the two axial momentum fluxes are positive quantities, $G_{z 1} \geq 0$ and $G_{z 2} \geq 0$, Eq. (6) yields the folllowing inequality for $C_{F}:-1 \leq C_{F} \leq G_{z 2} / G_{z 1}$. The evolution of the swirl number through a tube with a variable cross section area is controlled by the pressure force applied to the impermeable boundary in the axial direction through the ratio $C_{F}=F_{z} / G_{z 1}$ in Eq. (9). Since the azimuthal momentum $G_{\theta}$ remains unaltered for an inviscid flow along a duct, the swirl variation is driven by the rate of conversion of the initial axial momentum flux $G_{z 1}$ to the axial force $F_{z}$ exerted on the impermeable boundary.

Let consider the generic cases of a nozzle and a diffuser as sketched in Fig. 8. Equation (9) shows that the swirl number necessarily increases in the converging nozzle because $p_{\Sigma}>p_{\infty}$ and the ratio $C_{F}$ is negative. It results in an increase of the swirl number due both to $R_{1} / R_{2}>1$ and $\left(1+C_{F}\right)^{-1}>1$.

The case of the diverging cup shown on the right in Fig. 8 is more difficult to handle and does not lead to a systematic conclusion. The pressure distribution along the lateral wall now depends on the eventual presence of recirculation zones due to flow separation inside the diffuser. This pressure distribution is in this case much more sensitive to the exact geometry of the diffuser [28]. The pressure drop through the device results from a competition between the conversion of kinetic energy and pressures losses modeled here by a singular pressure loss coefficient $k$. In typical air swirling injectors, the head loss remains generally weak, and one seeks to keep $C_{F}$ as low as possible to limit pressure losses. This leads in Fig. 8 to a decrease in the swirl number between the inlet and the outlet sections of a diverging cup. The sign of $C_{F}$ in Fig. 8 is confirmed by the pressure measurements from Chigier and Beer [13].

In both the converging nozzle and diverging cup, the term $\left(1+C_{F}\right)^{-1}$ in Eq.(9) magnifies the respective increase and drop of swirl due to the change of the cross section area between the inlet and outlet. The contrast with Eq. (1) denotes that the swirl number evolution differs when taking into account the pressure terms. Note that the conservation of axial momentum flux reported in $[13,22]$ is interpreted here as $C_{F}$ being small in Eq. (9).

\section{Mechanical energy balance}

The previous qualitative analysis is deepened on a more quantitative basis with the help of shape factors that are defined as follows. Profiles are first set dimensionless with shape factors that characterize the inhomogeneous nature of the considered velocity profiles. Let $f_{z j}$ and $f_{\theta j}$ respectively designate the dimensionless profiles of the axial and tangential velocities, with $j=1,2$ :

$$
u_{z j}(r)=f_{z j}(r) U_{z j} \quad u_{\theta j}(r)=f_{\theta j}(r) U_{\theta j}
$$

where $U_{z j}$ and $U_{\theta j}$ are the area-averaged axial and tangential velocities shown in Fig. 8. They are defined as $U_{i j} A_{j}=\int_{A_{j}} u_{i j}(r) \mathrm{d} A$. The shape factor $f_{i j}(r)$ needs in turn to comply with $\int_{A_{j}} f_{i j}(r) \mathrm{d} A=A_{j}$, with $i=z, \theta$ and $j=1,2$.

One can express the pressure-less swirl variation through a change of the cross section area by:

$$
\frac{\widetilde{S}_{2}}{\widetilde{S}_{1}}=\frac{R_{2}}{R_{1}} \frac{\int_{A_{1}} f_{z 1}^{2}(r) \mathrm{d} A / A_{1}}{\int_{A_{2}} f_{z 2}^{2}(r) \mathrm{d} A / A_{2}}
$$

Due to the angular momentum conservation, change of the swirl number is fully controlled by the axial flow velocity profile at the system terminations. This expression generalizes Eq. (1) from Gupta and Lilley [4] established for a constant axial velocity $\left(f_{z 1}=f_{z 2}=1\right)$ to velocity profiles of arbitrary shapes obeying to Eq. (10).

Equation (11) is now used to highlight the impact of the structure of the velocity profiles on the evolution of the swirl number. The LDV measurements reported in Fig. (7) are used to determine the shape factors $f_{z 1}$ and $f_{z 2}$ at the diffuser outlet, for two different diffusers of respective radius $R_{1}$ and $R_{2}$. In doing so, one retrieves the experimental result $\widetilde{S}_{2} / \widetilde{S}_{1} \simeq 1$, as in Eq. (4). Therefore, the use of shape factors in Eq. (11) reconciliates the evolution of the swirl number Eq. (4) measured by LDV with the model Eq. (1) from Gupta and Lilley. This validation underlines that the value of the swirl number mainly relies on the assumptions made on the velocity profiles.

Shape factors are now used to close the dimensionless pressure force $C_{F}$ term in Eq. (9) by a balance of mechanical energy applied to the control volume delimited by the inlet $A_{1}$ and outlet $A_{2}$ sections of the diverging quarl. The flow is again considered as steady, with negligible viscous effects. The mechanical energy balance 
is expressed in its integral form:

$$
\begin{array}{r}
\int_{A_{1}}\left(\frac{1}{2} \rho \mathbf{v}^{2}+p\right) \mathbf{v} \cdot \mathbf{n} \mathrm{d} A-\int_{A_{2}}\left(\frac{1}{2} \rho \mathbf{v}^{2}+p\right) \mathbf{v} \cdot \mathbf{n} \mathrm{d} A \\
=k \int_{A_{1}} \frac{1}{2} \rho u_{z}^{2} \mathbf{v} \cdot \mathbf{n} \mathrm{d} A
\end{array}
$$

where $k$ denotes the head loss through the diverging quarl. Mass, momentum and energy balances are rewritten with the help of shape factors. Quantities are set dimensionless with respect to $U_{z 1}$. For a fixed ratio $U_{\theta 1} / U_{z 1}$ characterizing the angular velocity of the upstream flow, the set of three balance equations is solved to determine the ratios $U_{z 2} / U_{z 1}, U_{\theta 2} / U_{z 2}$ and $\left(p_{1}-p_{\infty}\right) /\left(\rho U_{z 1}^{2}\right)$. When assuming the shape factor distribution, the swirl number $S_{2} / S_{1}$ comes as a result.

An analytical solution is derived for a uniform axial flow $f_{z j}=1$ and a solid-body rotation $f_{\theta j}=(3 / 2)(r / R)$ at the inlet $(j=1)$ and outlet $(j=2)$ of the diffuser, as Gupta and Lilley [4] did in their model Eq. (1). The resolution of the system leads to the pressure coefficient $C_{p}=\left(p_{2}-p_{1}\right) /\left(\rho U_{z_{1}}^{2} / 2\right)$ and the pressure-dependent swirl number $S$ :

$$
C_{p}=\frac{p_{2}-p_{1}}{\rho U_{z 1}^{2} / 2}=\left(1-x^{-2}\right)\left(x^{-2}+1+2{\widetilde{S_{1}}}^{2}\right)-k
$$

and

$$
\frac{S_{2}}{S_{1}}=x\left[1+\frac{k}{2}+\frac{1}{2}\left(x^{-2}-1\right)\left(x^{-2}+1+2{\widetilde{S_{1}}}^{2}\right)\right]
$$

where $x=R_{2} / R_{1}$ and $\widetilde{S}_{1}=\frac{\Omega_{1} R_{1}}{2 U_{11}}$ designates the pressure-less swirl number, and $\Omega_{1}$ the angular velocity at the diffuser inlet. It appears that the ratio of the swirl number does not only depend on the ratio $R_{2} / R_{1}$ but also on the pressure-less swirl number $\widetilde{S}_{1}$ and head loss $k$. As the swirl evolution is not straightforward in Eq. (14), a Taylor expansion in the neighborhood of $R_{1}=R_{2}$ yields :

$$
\frac{S_{2}}{S_{1}}=1+\frac{k}{2}-\left(\frac{R_{2}}{R_{1}}-1\right)\left(1+2{\widetilde{S_{1}}}^{2}-\frac{k}{2}\right)
$$

This latter expression describes the decline of the swirl number through a small diverging cup.

Figure 9 depicts the swirl number evolution through a diffuser for different inlet swirl numbers $S_{1}$ in the absence of head loss $k=0$. The scope of these formula remains limited as the head loss $k$ needs to be specified and depends itself on several flow parameters. It is however shown here that the pressure contribution to the swirl number can be evaluated with a balance of mechanical energy. This energy budget shows that an increasing swirl level $\widetilde{S}_{1}$ at the diffuser inlet leads to a

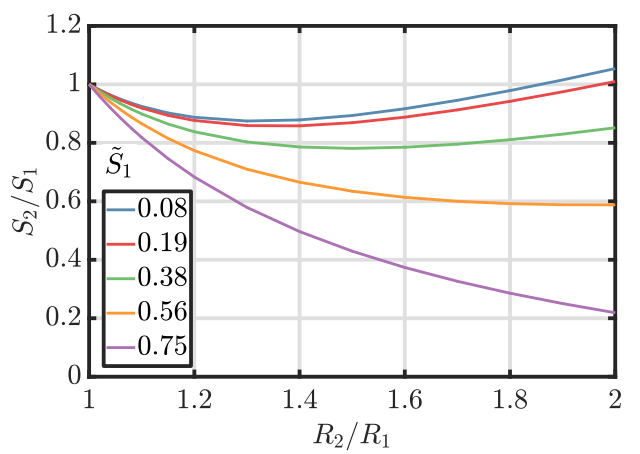

Figure 9: Swirl number ratio $S_{2} / S_{1}$ for different inlet pressure-less swirl number $S_{1}$, with $k=0$.

higher kinetic energy loss through a section change, so that $G_{z 1}$ decreases with $p_{1}-p_{2}$, yielding a smaller ratio $S_{2} / S_{1}$.

As a conclusion, the impact of the quarl angle on the swirl number $S$ evolution has been investigated from different perspectives: (i) through LDV measurements of the pressure-less swirl number $\widetilde{S}$, (ii) with a theoretical analysis of the swirl number $S$ evolution with the help of Eq. (9), (iii) with the help of shape factors to solve the mechanical energy balance in Eq. (14). It has been shown that in all three cases, the swirl number decreases with the quarl angle expansion, and that the pressure-less swirl number $\vec{S}$ is not altered through the cross section area change. This theoretical analysis confirms that changes of the swirl level $S$ through the injector diffuser cup, regardless the method used to evaluate this change, cannot explain the structure of the flame and flow patterns observed in the experiments when the injector cup angle is varied.

It is at this point worth recalling the assumptions made in the experimental and theoretical analysis carried out in this work. First, the expressions Eqs. (9) and (14) derived in this work result from inviscid theory, in which effects of turbulence have also been neglected. Measurements of the swirl number carried out in this work do not include effects of the turbulent velocity fluctuations either. Secondly, as the overall study focuses on the influence of the quarl angle, all other geometrical parameters that are known to alter the flame and flow patterns have been kept constant. For instance, effects of the injector geometry have been investigated in [24]. Effects of the combustion chamber confinement have been investigated in [9] and this study pertains to situations in which the confinement ratio is large. 


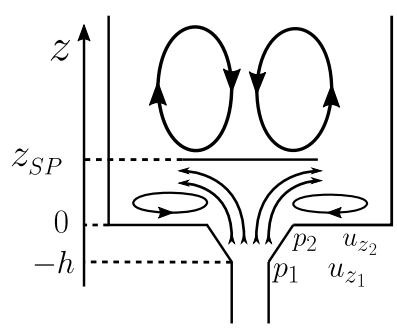

Figure 10: Schematic of the flow depicted as a stagnation flow between the injector quarl and the internal recirculation zone.

\section{Impact of quarl on the stagnation point position of the IRZ}

Measurements in Fig. 4 show that the growth of the IRZ is promoted by a large increase in the radial component of the flow when the quarl angle rises from $0^{\circ}$ up to $45^{\circ}$. Through the continuity equation, the gradient of radial velocity is balanced with the negative gradient of axial velocity in the vicinity of the diverging nozzle outlet. The following analysis is carried out so as to provide a model for the displacement of the position of the internal recirculation zone towards the injector outlet when the quarl angle increases.

Let assume that a swirling jet passes through a diverging quarl, with a sufficiently high level of swirl to create an inner recirculation zone in the combustion chamber, as sketched in Fig. 10. The pressures, velocities and cross section areas are indexed by 1 at the diffuser inlet, and by 2 at the outlet of the diverging cup. The quarl outlet also defines the axial origin, whereas $z_{S P}$ stands for the axial coordinate of the stagnation point defining the lower position of the IRZ along the burner axis.

The axial velocity gradient along the burner axis is set by the adverse pressure gradient, no matter the swirl motion :

$$
\frac{\partial p}{\partial z}=-\rho u_{z} \frac{\partial u_{z}}{\partial z}
$$

The adverse pressure gradient is promoted by the expansion of the quarl and stays positive up to the stagnation point $z_{S P}$. The momentum balance Eq. (16) is now evaluated at the quarl outlet $z=0$. Figure 5 indicates that the swirling jet opening angle $\beta$ regularly increases like the angle $\alpha$ of the diffuser cup as long as it lies in the free jet regime : $\beta \simeq \alpha$ when $\alpha<30^{\circ}$. Therefore, following the streamlines, the pressure gradient at the quarl outlet in section (2) is then equal to the pressure gradient at the quarl inlet in section (1) :

$$
\frac{p_{2}-p_{1}}{h} \sim-\left.\rho u_{z 2} \frac{\partial u_{z}}{\partial z}\right|_{z=0}
$$

The impact of the internal recirculation zone on the flow in the vicinity of the injector outlet can be considered as a typical stagnation flow with a strain rate $\epsilon$. The inviscid flow thus obeys to $u_{z}=u_{z 2}-\epsilon z$, and $z_{S P}=$ $u_{z 2} / \epsilon$. By eliminating $\epsilon$, the height of the stagnation point thus scales as:

$$
\frac{z_{S P}}{h} \sim\left(\frac{A_{1}}{A_{2}}\right)^{2} \frac{1}{C_{p}} \quad \text { with } \quad C_{p}=\frac{p_{2}-p_{1}}{\rho U_{z 1}^{2}}
$$

The pressure coefficient $C_{p}$ is given by Eq. (13) showing that a diverging quarl increases the pressure drop $C_{p}$ positively. Equation (19) states that the adverse pressure gradient moves the stagnation point $z_{S P}$ further upstream closer to the injector outlet due to the cross section area ratio $\left(A_{1} / A_{2}\right)^{2}$ and the inverse of the pressure coefficient $1 / C_{p}$ that both decrease for increasing quarl angles.

This behavior is precisely the one which is observed in Figs. 4 and 5. Predictions from Eq. (19) are superimposed to the measurements of the IZR leading edge position along the burner axis in Fig. 5. To do so, the values found for $\widetilde{S}=0.85$ and $k=0.5$ are used to determine $C_{p}$ with Eq. (13) and the model is calibrated with the measurements made for $\alpha=10^{\circ}$. No difference has been found when changing the head loss coefficient from $k=0$ up to $k=1$ because the major contribution in Eq. (19) lies in the area ratio $A_{1} / A_{2}$. The match between the model and the measurements is excellent in Fig. 5, except in the absence of quarl when $\alpha=0^{\circ}$. In this case, the adverse pressure gradient in Eq. (16) is not related to the quarl, but to the natural expansion of the swirling jet, a feature which is not taken into account in the present analysis.

It has been shown that the position of the leading edge front of a swirling flame can be controlled in the Oxytec test-rig by adjusting the angle of the diffuser cup from the injector. It has then been demonstrated that the resulting modification of the swirl level due to the quarl does not take part in the process because the diverging cup reduces the swirl number $S$ as seen in Eq. (4) and let the pressure-less swirl number $\widetilde{S}$ unaltered (Eq. (9)) in the Oxytec test-rig. It has then been shown that the axial velocity gradient at the injector outlet mainly depends on both the magnitude of the axial velocity and the pressure drop induced by the quarl. The quarl expansion is responsible for both a stronger adverse pressure gradient and a reduction of the axial flow velocity.

Though assuming a stagnation flow pattern at the burner outlet constitutes a rough approximation of reality, the model Eq. (17) developed in this study successfully reproduces the evolution of the stagnation point position $z_{S P}$ of the internal recirculation region as a function of the axial velocity gradient at the burner outlet $\partial u_{z} / \partial z(z=0)$ as observed in the experiments. This 
physics based model may be used as a starting point to develop more realistic representations of the swirled flow at the outlet of a swirling injector and needs to be further corroborated with other injector technologies.

$$
\frac{z_{S P}}{h} \sim\left(\frac{A_{20}}{A_{2}}\right)^{2} \frac{1}{J} \frac{1}{C_{p}} \quad \text { with } \quad C_{p}=\frac{p_{2}-p_{1}}{\rho U_{z 1}{ }^{2}}
$$

\section{CONCLUSION}

The impact of a diverging cup on the structure of technically premixed swirling flames has been investigated experimentally and analytically. In this study, flames are stabilized aerodynamically at the injector outlet of the flow produced by an axial-plus-tangential swirler ended by a diffuser cup with an adjustable angle.

Flame topologies have been observed with $\mathrm{OH}^{*}$ chemiluminescence imaging. PIV and OH PLIF measurements in reacting conditions have provided information on the structure of the flame and the internal recirculation zone produced by the swirled flow. For a given geometrical swirl number calculated before the diffuser cup, increasing the quarl angle considerably widens the internal recirculation zone, shortens the flame and moves the position of the IRZ upstream closer to the burner outlet. A large value of the quarl angle can place the flow and flame patterns in the wall-jet regime, at a confinement ratio where a free-jet regime is generally produced in the absence of quarl.

A comparison with non reacting flow conditions has shown a very similar evolution of the position of the internal recirculation region as the quarl angle widens with results obtained under reacting conditions. LDV has been carried out to determine the swirl number without pressure terms for the different quarl angles tested. It has been found that the pressure-less swirl number $S$ remains unaltered by the quarl expansion, a result which conflicts with the predictions from Gupta and Lilley et al. [4]. The measured swirl levels $\widetilde{S}$ are therefore seen not to account for the drastic increase of the internal recirculation as the quarl angle increases.

A theoretical analysis has been carried out to take the pressure contribution into account in the Swirl number $S$ and examine the impact of a diverging cup on the swirl number evolution. It has been found that the swirl number $S$ decreases as the quarl angle increases, and that this trend is magnified when pressure effects are included. The pressure contribution reduces the upstream axial momentum flux. Hence, neither the measured pressure-less swirl number nor the theoretical estimates allow to account for the flame and flow patterns observed in the experiments. It is firmly concluded that the swirl number is not the relevant dimensionless quantity to assess the impact of a nozzle cup on the flame and flow patterns when the quarl angle is varied, all other parameters remaining fixed.

It is finally found that the decrease of the axial flow velocity and increase of the adverse pressure gradient at the burner outlet are both responsible for the displacement of the position of the stagnation point of the internal recirculation zone as the quarl angle increases. The diffuser expansion is seen to diminish the axial momentum flux per unit area, which reduces the jet ability to push the internal recirculation zone further downstream. A theoretical model has been developed that well reproduces the experimental data for the diffuser cup angles tested between $5^{\circ} \leq \alpha \leq 30^{\circ}$ by assuming that the swirling flow takes the structure of a stagnation flow at the burner outlet.

\section{ACKNOWLEDGMENTS}

This work is supported by the Air Liquide, CentraleSupélec and CNRS Chair on oxy-combustion and heat transfer for energy and environment and by the OXYTEC project (ANR-12-CHIN- 0001) from l'Agence Nationale de la Recherche. We also would like to thank the technical staff of EM2C for their assistance during the design and construction of the experimental setup.

[1] Rawe, R., and Kremer, H., 1981. "Eighteenth symposium (international) on combustion stability limits of natural gas diffusion flames with swirl". Symposium (International) on Combustion, 18(1), pp. $667-677$.

[2] Syred, N., 2006. "A review of oscillation mechanisms and the role of the precessing vortex core (pvc) in swirl combustion systems". Progress in Energy and Combustion Science, 32(2), pp. $93-161$.

[3] Beér, J. M., and Chigier, N. A., 1972. "Combustion aerodynamics". New York.

[4] Gupta A. K., Lilley D. G., S. N., 1984. Swirl flows. Abacus Press, Tunbridge Wells, Kent, England.

[5] Cheng, R., Yegian, D., Miyasato, M., Samuelsen, G., Benson, C., Pellizzari, R., and Loftus, P., 2000. "Scaling and development of low-swirl burners for low-emission furnaces and boilers". Proceedings of the Combustion Institute, 28(1), pp. 1305 $-1313$.

[6] Chterev, I., Sundararajan, G., Seitzman, J., and Lieuwen, T., 2015. "Precession effects on the relationship between timeaveraged and instantaneous swirl flow and flame characteristics". In ASME Turbo Expo 2015, GT2015-42768.

[7] Burmberger, S., Hirsch, C., and Sattelmayer, T., 2006. "Designing a radial swirler vortex breakdown burner". In ASME Turbo Expo 2006, GT2006-90497.

[8] Toh, K., Honnery, D., and Soria, J., 2010. "Axial plus tangential entry swirling jet”. Experiments in Fluids, 48(2), pp. 309-325.

[9] Fanaca, D., Alemela, P., Hirsch, C., and Sattelmayer, T., 2010. "Comparison of the flow field of a swirl stabilized premixed 
burner in an annular and a single burner combustion chamber". Journal of Engineering for Gas Turbines and Power, 132(7), p. 071502.

[10] Mongia, H., 2011. "Engineering aspects of complex gas turbine combustion mixers part iii: 30 opr". In 9th Annual International Energy Conversion Engineering Conference, p. 5525.

[11] Chong, L. T. W., Komarek, T., Zellhuber, M., Lenz, J., Hirsch, C., and Polifke, W., 2009. "Influence of strain and heat loss on flame stabilization in a non-adiabatic combustor". In the European Combustion Meeting.

[12] Guiberti, T., Durox, D., Scouflaire, P., and Schuller, T., 2015. "Impact of heat loss and hydrogen enrichment on the shape of confined swirling flames". Proceedings of the Combustion Institute, 35(2), pp. 1385 - 1392.

[13] Chigier, N. A., and Beér, J. M., 1964. "Velocity and staticpressure distributions in swirling air jets issuing from annular and divergent nozzles". Journal of Basic Engineering, 86(4), pp. 788-796.

[14] Terhaar, S., Oberleithner, K., and Paschereit, C. O., 2014. "Impact of steam-dilution on the flame shape and coherent structures in swirl-stabilized combustors". Combustion Science and Technology, 186(7), pp. 889-911.

[15] Vanoverberghe, K. P., Bulck, E. V. V. D., and Tummers, M. J., 2003. "Confined annular swirling jet combustion". Combustion Science and Technology, 175(3), pp. 545-578.

[16] Therkelsen, P. L., Littlejohn, D., Cheng, R. K., Portillo, J. E., and Martin, S. M., 2010. "Effect of combustor inlet geometry on acoustic signature and flow field behavior of the low swirl injector". In ASME Turbo Expo 2010, GT2010-23498, pp. 11831194.

[17] Jourdaine, P., Mirat, C., Beaunier, J., Caudal, J., Joumani, Y., and Schuller, T., 2016. "Effect of quarl on N2- and CO2-diluted methane oxy-flames stabilized by an axial-plustangential swirler". In ASME Turbo Expo. GT2016-56953.

[18] Weber, R., and Dugué, J., 1992. "Combustion accelerated swirling flows in high confinements". Progress in Energy and Combustion Science, 18(4), pp. 349-367.

[19] Syred, N., and Bekr, J. M., 1974. "Combustion in Swirling Flows : A FReview”. Combustion and Flame, 201(2), pp. 143201.

[20] Mattingly, J. D., Oates, G. C., Dolling, D. S., and Gray, W. K., 1986. "An experimental investigation of the mixing of coannular swirling flows". AIAA Journal, 24(5), pp. 785-792.

[21] Dixon, T. F., Truelove, J. S., and Wall, T. F., 1983. "Aerodynamic Studies on Swirled Coaxial Jets from Nozzles with Divergent Quarls". J. Fluid Eng., 105(2), pp. 197-203.

[22] Mahmud, T., Truelove, J. S., and Wall, T. F., 1987. "Flow Characteristics of Swirling Coaxial Jets From Divergent Nozzles". Journal of Fluids Engineering, 109 (September 1987), pp. 275282.

[23] Jourdaine, P., Mirat, C., Caudal, J., Lo, A., and Schuller, T., 2017. "A comparison between the stabilization of premixed swirling co 2-diluted methane oxy-flames and methane/air flames". Fuel, 201, pp. 156-164.

[24] Jourdaine, P., Mirat, C., Caudal, J., and Schuller, T., 2017. "Stabilization mechanisms of swirling premixed flames with an axial-plus-tangential swirler". In ASME Turbo Expo, GT201764248.

[25] Guiberti, T. F., Durox, D., Zimmer, L., and Schuller, T., 2015. "Analysis of topology transitions of swirl flames interacting with the combustor side wall". Combustion and Flame, 162(11), pp. $4342-4357$.

[26] Lefebvre, A. H., 2010. Gas turbine combustion: alternative fuels and emissions. CRC press.

[27] Reichel, T. G., Terhaar, S., and Paschereit, O., 2015. "Increasing flashback resistance in lean premixed swirl-stabilized hydrogen combustion by axial air injection". Journal of Engineering for Gas Turbines and Power, 137(7), p. 071503.

[28] Schlichting, H., Gersten, K., Krause, E., and Oertel, H., 1955. Boundary-layer theory, Vol. 7. Springer. 